\title{
Reliable Prediction of Insulin Resistance by a School-Based Fitness Test in Middle-School Children
}

\author{
Todd Varness, ${ }^{1}$ Aaron L. Carrel, ${ }^{1}$ Jens C. Eickhoff, ${ }^{2}$ and David B. Allen${ }^{1}$ \\ ${ }^{1}$ Department of Pediatrics, University of Wisconsin School of Medicine and Public Health, H4/4 Clinical Science Center, \\ 600 Highland Avenue, Madison, WI 53792-4108, USA \\ ${ }^{2}$ Department of Statistics, Colorado State University, 206 Statistics Building, Fort Collins, CO 80523, USA
}

Correspondence should be addressed to Todd Varness, tvarness@pediatrics.wisc.edu

Received 11 May 2009; Accepted 24 July 2009

Recommended by Myron Genel

Objectives. (1) Determine the predictive value of a school-based test of cardiovascular fitness (CVF) for insulin resistance (IR); (2) compare a "school-based" prediction of IR to a "laboratory-based" prediction, using various measures of fitness and body composition. Methods. Middle school children $(n=82)$ performed the Progressive Aerobic Cardiovascular Endurance Run (PACER), a school-based CVF test, and underwent evaluation of maximal oxygen consumption treadmill testing $\left(\mathrm{VO}_{2}\right.$ max), body composition (percent body fat and BMI $z$ score), and IR (derived homeostasis model assessment index [HOMA $[$ IR $]$ ). Results. PACER showed a strong correlation with $\mathrm{VO}_{2} \mathrm{max} / \mathrm{kg}\left(r_{s}=0.83, P<.001\right)$ and with $\mathrm{HOMA}_{\mathrm{IR}}\left(r_{s}=-0.60, P<.001\right)$. Multivariate regression analysis revealed that a school-based model (using PACER and BMI $z$ score) predicted IR similar to a laboratory-based model (using $\mathrm{VO}_{2} \mathrm{max} / \mathrm{kg}$ of lean body mass and percent body fat). Conclusions. The PACER is a valid school-based test of CVF, is predictive of IR, and has a similar relationship to IR when compared to complex laboratory-based testing. Simple school-based measures of childhood fitness (PACER) and fatness (BMI $z$ score) could be used to identify childhood risk for IR and evaluate interventions.

Copyright () 2009 Todd Varness et al. This is an open access article distributed under the Creative Commons Attribution License, which permits unrestricted use, distribution, and reproduction in any medium, provided the original work is properly cited.

\section{Introduction}

Insulin Resistance (IR) is an independent predictor for the development of hypertension, coronary heart disease, stroke, cancer, and type 2 diabetes, and greater insulin sensitivity is a protective factor against these clinical conditions [1]. IR and type 2 diabetes are increasing in childhood and adolescence [2].

In addition to obesity and genetic predisposition, poor cardiovascular fitness (CVF) is an important independent risk factor for IR [3]. Poor CVF increases a person's risk for cardiovascular disease, hypertension, and type 2 diabetes [46], and improved CVF attenuates the morbidities associated with obesity. As a result, active yet overweight individuals can have lower risk for type 2 diabetes and cardiovascular disease than sedentary normal-weight individuals $[7,8]$. Thus, an important public health goal is the improvement of CVF in the population in general, not just obese persons.

Unfortunately, the current gold standard for assessing CVF, maximal oxygen consumption treadmill or bike testing
$\left(\mathrm{VO}_{2} \max \right)$, requires specialized equipment, time, and expert supervision, and is therefore not practical for evaluation of large groups of children in school or community settings. To expand the ability to identify children with IR, a feasible school-based test of childhood CVF shown to be predictive of IR is needed.

FITNESSGRAM, developed in 1982 by The Cooper Institute, is a validated battery of tests that can be used to assess childhood physical fitness in schools. The Progressive Aerobic Cardiovascular Endurance Run (PACER) is a component of the FITNESSGRAM and consists of a multistage progressive 20-meter shuttle test (20MST). The PACER is a valid school-based test of CVF in pediatric populations [9]. Although laboratory-based fitness assessment $\left(\mathrm{VO}_{2} \max \right)$ has been shown to be predictive of IR, it is not known if a school-based fitness assessment such as the PACER is sufficiently accurate to be predictive of IR.

The objectives of this study are (1) determine the predictive value of the PACER, alone and controlled for body composition, for IR; (2) compare "school-based" and 
"laboratory-based" assessments of childhood fitness and body composition in terms of predicting IR.

\section{Materials and Methods}

Children $(n=82)$ from two local middle schools participated in this study. Over a 12-month period (fall of 2006 to fall of 2007), each participant underwent testing at the University of Wisconsin Exercise Science Laboratory after an overnight fast. The procedures were approved by the Human Subjects Committee, and informed written consent was obtained before initiating the testing protocol. Testing included a physical examination, blood work, body composition assessment, and CVF assessment. Within 14 days of this testing, the participants performed the PACER at their schools. Subjects with overt type 2 diabetes were excluded.

Percent body fat (\%BF) and lean body mass (LBM) were measured by dual-energy X-ray absorptiometry (DXA). Whole body scans were performed with the Norland XR36 whole body bone densitometer (Norland Corporation, Ft. Atkinson, WI) following standard calibration, and tissue masses were analyzed with software version 3.7.4/2.1.0. Height was measured on a wall-mounted stadiometer to the nearest $0.5 \mathrm{~cm}$. Weight was measured on a calibrated beam balance platform scale to the nearest $0.1 \mathrm{~kg}$. "Obese" was defined as a BMI $\geq$ 95th percentile for age and gender.

The gold standard CVF assessment was maximal oxygen consumption $\left(\mathrm{VO}_{2} \max \right)$, determined by open-circuit spirometry using a progressive treadmill walking protocol to volitional fatigue with a Medical Graphics CPX-D treadmill (St. Paul, MN). The speed of the treadmill was set initially per the subject's comfort, starting at $0 \%$ grade and increasing $2 \%$ every minute. Requirements to strictly define whether subjects reached their maximal oxygen consumption by this protocol included at least 2 of the following three criteria: (1) maximal heart rate $>200$ beats per minute; (2) respiratory exchange ratio $\left(\mathrm{VCO}_{2} / \mathrm{VO}_{2}\right)>1$; and (3) a plateau in oxygen consumption. All of the children included in the data analysis met at least 2 of the 3 criteria. $\mathrm{VO}_{2}$ max can be expressed as $\mathrm{VO}_{2}$ max per $\mathrm{kg}$ of total body weight $\left(\mathrm{VO}_{2} \max / \mathrm{kg}\right)$ or as $\mathrm{VO}_{2}$ max per $\mathrm{kg}$ of LBM $\left(\mathrm{VO}_{2} \mathrm{max} / \mathrm{kgLBM}\right)$. Since the best measurement of fitness is still debated, we analyze our data using both measures of CVF.

The measure of IR for this study was HOMA IR. HOMA IR is a validated equation that adjusts the fasting insulin and glucose to provide a reliable assessment of insulin resistance, particularly in obese children and adolescents $[10,11]$. In children, the HOMA $(r=0.91)$ correlates closely with euglycemic clamp studies [11]. $\mathrm{HOMA}_{\mathrm{IR}}$ was calculated with the formula: fasting insulin $(\mu \mathrm{IU} / \mathrm{mL}) \times$ fasting glucose $(\mathrm{mmol} / \mathrm{L}) / 22.5$. Fasting insulin concentration was determined with the chemiluminescent assay (ARUP Laboratories, Salt Lake City, UT), and glucose concentration was determined by an enzymatic method (Beckman Diagnostics, Fullerton, CA). According to ARUP Laboratories, at a mean insulin level of $14.8 \mu \mathrm{IU} / \mathrm{mL}$, the standard deviation of the insulin assay is 1 and the coefficient of variation is $6.7 \%$.

The PACER was conducted by school physical education teachers trained in administering the tests according to the FITNESSGRAM protocol [9]. The PACER is a multistage progressive 20-meter shuttle run. Subjects run back and forth along a 20 -meter course, and each minute the pace required to run the 20 meters increases. The pace is determined by beeps from a pre-recorded tape or $\mathrm{CD}$. The initial running speed is $8.5 \mathrm{~km} /$ hour, and the speed increases by $0.5 \mathrm{~km} / \mathrm{h}$ every minute. The test is finished when the subject fails to complete the 20-meter run in the allotted time twice [9]. Because the number of laps per minute increases as the running speed increases, the PACER results are "scored" as number of laps successfully completed. The PACER results were also analyzed using running speed at the last completed one-minute stage and the estimated $\mathrm{VO}_{2} \mathrm{max} / \mathrm{kg}$ using the Leger et al. equation, which is the regression formula utilized by the FITNESSGRAM software [12]. The Leger equation is estimated $\mathrm{VO}_{2} \max / \mathrm{kg}=31.025$ +3.238 (running speed in $\mathrm{km} / \mathrm{hr}$ ) -3.248 (age in years) +0.1536 (running speed $\times$ age) [12]. In the regression analysis, the PACER is expressed as number of laps completed.

Body composition, fitness, and insulin resistance measurements were summarized by standard descriptive statistics in terms of mean \pm SD. The comparisons of all continuous measurements between subgroups (males versus females) were performed using a two-sample $t$-test or nonparametric Wilcoxon rank sum test, when data were not normally distributed. The univariate associations between fitness and body composition variables and $\mathrm{HOMA}_{\mathrm{IR}}$ were examined with the nonparametric Spearman rank correlation analysis. Multivariate linear regression analysis was performed for both a school-based model (with PACER as a measure of fitness and BMI $z$ score as a measure of body composition) and a laboratory-based model (with $\mathrm{VO}_{2} \mathrm{max} / \mathrm{kgLBM}$ as a measure of fitness and $\% \mathrm{BF}$ by DXA as a measure of body composition) to assess the independent contributions of fitness and body composition on insulin resistance. Both models were controlled for age and gender. The $\mathrm{HOMA}_{\mathrm{IR}}$ was log-transformed in the regression analyses to meet the assumption of normality. Variance inflation factors were calculated for each multivariate regression model in order to assess for collinearity. Standard model diagnostic tools (residual plots, etc.) were used to examine model assumptions. The predictive power of the regression models was assessed by performing cross-validation analysis. Cross-validation studies were conducted by randomly selecting sub-datasets which were used to fit the model and then validated on the remaining data. This was repeated 1000 times, and the root mean square error (RMSE) across all 1000 replications of the predicted versus observed values was calculated. A smaller RMSE indicates better prediction. All statistical analyses were performed with SAS software (version 9.1; SAS Institute, Cary, NC). All $P$ values were 2 -sided, and $P$ values $<.05$ were considered statistically significant. 
TABLE 1: Study subject characteristics $(n=82)$.

\begin{tabular}{|c|c|c|c|c|}
\hline & Total & Males & Females & $P$-value ${ }^{\dagger}$ \\
\hline \multicolumn{5}{|l|}{ Descriptive characteristics } \\
\hline Number & 82 & 34 & 48 & \\
\hline Age (years) & $13.0 \pm 0.7$ & $13.1 \pm 0.7$ & $12.9 \pm 0.7$ & .5028 \\
\hline Height $(\mathrm{cm})$ & $162.8 \pm 7.7$ & $162.2 \pm 8.2$ & $163.3 \pm 7.3$ & .2958 \\
\hline Weight (kg) & $65.0 \pm 19.7$ & $67.5 \pm 19.9$ & $63.3 \pm 19.5$ & .3094 \\
\hline Obese (BMI $\geq 95$ th percentile) & $26(32 \%)$ & $17(50 \%)$ & $9(19 \%)$ & $.0027^{*}$ \\
\hline \multicolumn{5}{|l|}{ Body composition assessments } \\
\hline BMI & $24.4 \pm 6.5$ & $25.5 \pm 6.6$ & $23.5 \pm 6.3$ & .2193 \\
\hline BMI $z$ score & $1.0 \pm 1.1$ & $1.2 \pm 1.2$ & $0.8 \pm 1.1$ & .1163 \\
\hline Percent body fat & $28.8 \pm 8.6$ & $28.3 \pm 10.9$ & $29.0 \pm 6.7$ & .7775 \\
\hline Lean body mass $(\mathrm{kg})$ & $37.41 \pm 8.86$ & $39.47 \pm 9.12$ & $36.00 \pm 8.48$ & .0778 \\
\hline \multicolumn{5}{|l|}{ CVF assessments } \\
\hline $\mathrm{VO}_{2} \max (\mathrm{mL} / \mathrm{kg} / \mathrm{min})$ & $40.4 \pm 9.4$ & $43.4 \pm 11.3$ & $38.3 \pm 7.1$ & $.0239^{*}$ \\
\hline $\mathrm{VO}_{2} \max / \mathrm{kgLBM}(\mathrm{mL} / \mathrm{kg} / \mathrm{min})$ & $67.8 \pm 8.2$ & $71.0 \pm 7.1$ & $65.5 \pm 8.2$ & $.0033^{*}$ \\
\hline PACER (laps) & $25.2 \pm 15.1$ & $27.7 \pm 17.7$ & $23.5 \pm 12.8$ & .2446 \\
\hline PACER speed $(\mathrm{km} / \mathrm{h})$ & $9.4 \pm 0.8$ & $9.5 \pm 0.9$ & $9.3 \pm 0.7$ & .2505 \\
\hline Estimated $\mathrm{VO}_{2} \max (\mathrm{mL} / \mathrm{kg} / \mathrm{min})[12]$ & $37.9 \pm 4.5$ & $38.4 \pm 4.9$ & $37.5 \pm 4.2$ & .3740 \\
\hline \multicolumn{5}{|l|}{ IR assessments } \\
\hline Fasting insulin $(\mu \mathrm{IU} / \mathrm{mL})$ & $15.0 \pm 8.0$ & $15.2 \pm 9.5$ & $14.9 \pm 6.8$ & .8419 \\
\hline Fasting glucose (mg/dL) & $92.8 \pm 6.8$ & $95.3 \pm 6.5$ & $91.2 \pm 6.5$ & $.0071^{*}$ \\
\hline $\mathrm{HOMA}_{\mathrm{IR}}$ & $3.5 \pm 1.9$ & $3.7 \pm 2.3$ & $3.4 \pm 1.6$ & .2766 \\
\hline
\end{tabular}

${ }^{\dagger}$ Comparison between males and females.

${ }^{*} P<.05$.

\section{Results}

3.1. Subject Characteristics. Subject characteristics are presented in Table 1 as mean \pm SD or number (percentage), and the $P$ value reflects the comparison between males and females. Seventeen of the 34 boys (50\%) and 9 of the 48 girls $(19 \%)$ had a BMI greater than the 95th percentile $(P=.003)$. Regardless of how CVF is expressed (relative to total body weight or lean body mass), boys had a higher CVF than girls. Girls had a higher fasting glucose level than boys (95.3 versus 93.8, $P=.007$ ), but no significant difference was seen between boys and girls in the fasting insulin or $\mathrm{HOMA}_{\mathrm{IR}}$.

3.2. PACER Validation. The PACER correlated significantly and closely with $\mathrm{VO}_{2} \max / \mathrm{kg}\left(r_{s}=0.83,95 \%\right.$ CI 0.74 to 0.93 , $P<.001$; Figure 1). The PACER also correlated significantly with $\mathrm{VO}_{2} \mathrm{max} / \mathrm{kgLBM}\left(r_{s}=0.55,95 \% \mathrm{CI} 0.37\right.$ to 0.68 , $P<.001)$. Since previous validation studies have compared the PACER to $\mathrm{VO}_{2} \max$, and not to $\mathrm{VO}_{2} \max / \mathrm{kgLBM}$, we used $\mathrm{VO}_{2} \mathrm{max} / \mathrm{kg}$ for the additional correlations between the PACER and CVF. In the 26 obese children with a BMI greater than the 95th percentile, the PACER was highly correlated with $\mathrm{VO}_{2} \mathrm{max} / \mathrm{kg}\left(r_{s}=0.74,95 \% \mathrm{CI} 0.49\right.$ to $\left.0.87, P<.001\right)$.

The PACER test result has been expressed in multiple ways in the literature. $\mathrm{VO}_{2} \mathrm{max} / \mathrm{kg}$ correlated strongly with

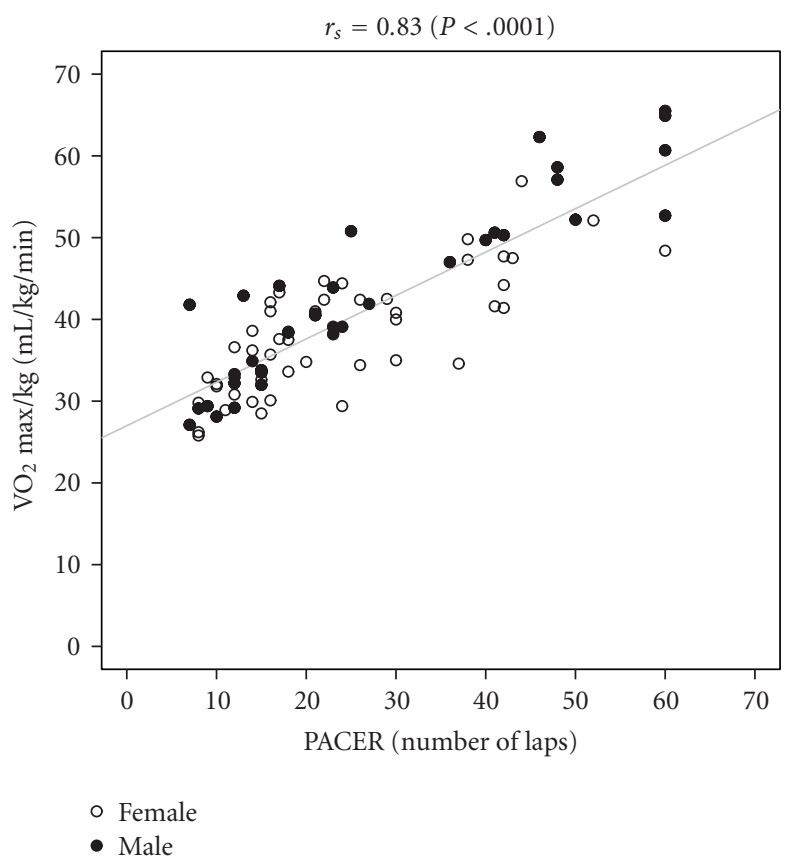

FIGURe 1: Relationship between PACER (number of laps) and $\mathrm{VO}_{2}$ $\max / \mathrm{kg}(\mathrm{mL} / \mathrm{kg} / \mathrm{min})$ in a sample of middle school children $(n=$ $82)$. 
TABLE 2: Univariate analysis for $\mathrm{HOMA}_{\mathrm{IR}}$ as a dependent variable $(n=82)$.

\begin{tabular}{|c|c|c|c|c|}
\hline Independent variable & $\beta($ se $)$ & $P$ value $^{+}$ & $r_{s}^{\dagger}$ & $\mathrm{RMSE}^{* *}$ \\
\hline PACER (laps) & $-0.07(0.01)$ & $<.0001^{*}$ & -0.60 & 1.68 \\
\hline PACER-estimated $\mathrm{VO}_{2} \max$ & $-0.21(0.04)$ & $<.0001^{*}$ & -0.51 & 1.72 \\
\hline $\mathrm{VO}_{2} \max$ & $-0.13(0.02)$ & $<.0001^{*}$ & -0.70 & 1.56 \\
\hline $\mathrm{VO}_{2} \max / \mathrm{kgLBM}$ & $-0.10(0.02)$ & $.0001^{*}$ & -0.47 & 1.79 \\
\hline BMI & $0.19(0.02)$ & $<.0001^{*}$ & 0.65 & 1.54 \\
\hline BMI $z$ score & $1.03(0.25)$ & $<.0001^{*}$ & 0.66 & 1.59 \\
\hline Percent body fat & $0.14(0.02)$ & $<.0001^{*}$ & 0.71 & 1.47 \\
\hline
\end{tabular}

${ }^{+} P$-value for testing $H_{0}: \beta=0$ versus $H_{A}: \beta \neq 0$.

† Spearman's rank correlation coefficient.

$* *$ Cross-validation Root Mean Square Error (RMSE) on original scale for $m=1000$ re-samples with sample size $n_{1}=50$ (model fitting) and $n_{2}=32$ ( $m o d e l$ validation).

$* P<.05$.

both the running speed $(\mathrm{km} / \mathrm{hr})$ at the last completed oneminute PACER stage $\left(r_{s}=0.83,95 \%\right.$ CI $\left.0.74-0.88, P<.001\right)$ and the estimated $\mathrm{VO}_{2} \mathrm{max} / \mathrm{kg}\left(r_{s}=0.83,95 \%\right.$ CI $0.75-$ $0.89, P<.001)$. Therefore, regardless of how the PACER is expressed (laps, running speed, or estimated $\mathrm{VO}_{2} \max$ ), there was a strong correlation between the PACER and laboratorybased CVF assessment.

3.3. Univariate Analysis. Table 2 shows the results of the univariate analysis between $\mathrm{HOMA}_{\mathrm{IR}}$ and all the variables of interest. The PACER was significantly negatively correlated with $\mathrm{HOMA}_{\mathrm{IR}}\left(r_{s}=-0.60,95 \% \mathrm{CI}-0.75\right.$ to $-0.44, P<.001$; Figure 2). Laboratory-based fitness assessments and the body composition assessments were also significantly and strongly correlated with $\mathrm{HOMA}_{\mathrm{IR}}$. An analysis using fasting insulin as the dependent variable revealed virtually identical results (data not shown), and therefore we focused additional analyses on $\mathrm{HOMA}_{\mathrm{IR}}$ only.

3.4. Multivariate Analysis. We used multivariate regression analysis to assess various measurements of fitness and body composition with $\mathrm{HOMA}_{\mathrm{IR}}$ as the dependent variable (Table 3). Both regression models were adjusted for sex and age. Model 1 utilized laboratory-based measurements of fitness $\left(\mathrm{VO}_{2}\right.$ max/kgLBM by maximal treadmill testing) and body composition (\%BF by DXA) on insulin resistance $\left(\mathrm{HOMA}_{\mathrm{IR}}\right)$. Since more recent studies investigating the relationship between CVF and IR used $\mathrm{VO}_{2} \mathrm{max} / \mathrm{kgLBM}$, we use $\mathrm{VO}_{2}$ max/kgLBM as the measure of fitness in the multivariate regression analysis. When we used $\mathrm{VO}_{2} \max / \mathrm{kg}$ as the measure of fitness, there was no difference in the results (data not shown).

The laboratory-based method (Model 1) accounted for $57 \%$ of the variance in $\mathrm{HOMA}_{\mathrm{IR}}$. Both $\mathrm{VO}_{2} \mathrm{max} / \mathrm{kgLBM}$ $(P<.001)$ and $\% \mathrm{BF}(P<.001)$ were significant independent predictors of insulin resistance by $\mathrm{HOMA}_{\mathrm{IR}}$. Model 2 utilized school-based measurements of fitness (PACER) and body composition (BMI $z$ score) on the HOMA IR. This school-based method accounted for $45 \%$ of the variance

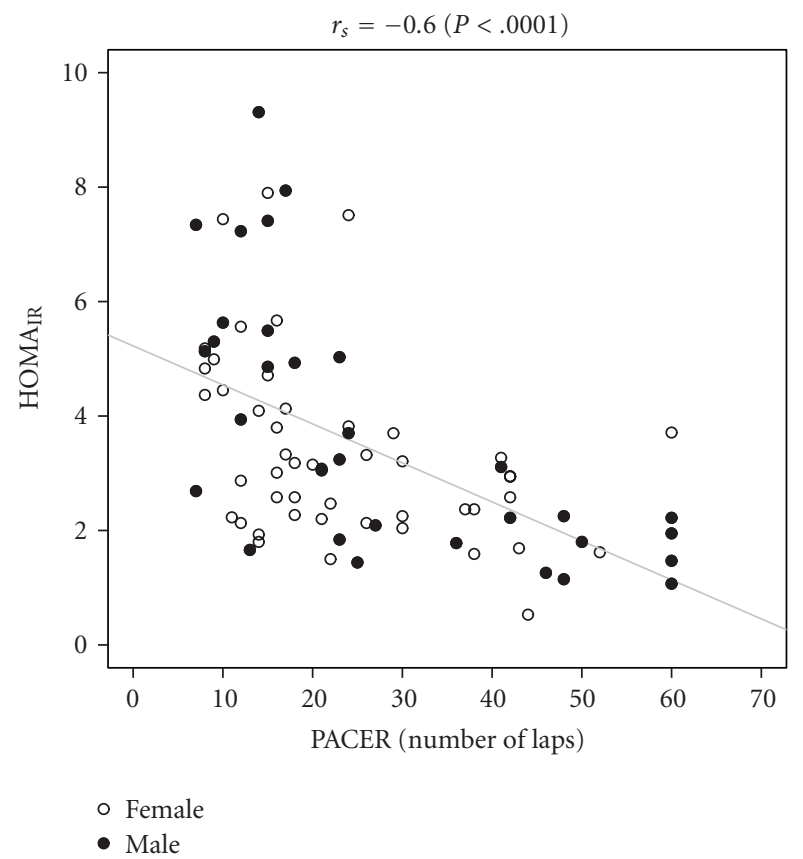

Figure 2: Relationship between PACER (number of laps) and $\mathrm{HOMA}_{\mathrm{IR}}$ in a sample of middle school children $(n=82)$.

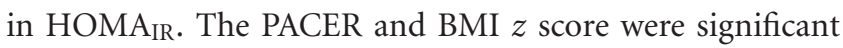
independent predictors of $\mathrm{HOMA}_{\mathrm{IR}}$, with slope parameters of $-0.012(P=.005)$ and $0.201(P<.001)$, respectively. In both multivariate regression models, the variance inflation factors were less than 2.5 , which indicate that colinearity did not confound the results.

The ability of the models to predict IR can be compared with the RMSE (a smaller RMSE indicates greater predictive value). The laboratory-based assessment of fitness and body composition (Model 1) had a slightly lower RMSE of 1.42 compared to an RMSE of 1.49 for the school-based assessment (Model 2). Both models had greater predictive value than using BMI $z$ score alone (RMSE of 1.59). Percent body fat, as determined by a DXA scan, had an RMSE of 1.47 . 
TAble 3: Regression coefficients $(\beta), P$ value, and coefficient of determination $\left(R^{2}\right)$ for the association of log $\left(\mathrm{HOMA}_{\mathrm{IR}}\right)$ with CVF and body composition in 2 multiple regression models, after adjusting for sex and age.

\begin{tabular}{|c|c|c|c|c|c|}
\hline & Variables & $\beta(\mathrm{se})$ & $P$ value $^{+}$ & $R^{2}$ & RMSE** $^{*}$ \\
\hline \multirow{2}{*}{ Model 1} & $\mathrm{VO}_{2} \max / \mathrm{kgLBM}$ & $-0.027(0.006)$ & $<.001^{*}$ & \multirow{2}{*}{0.57} & \multirow{2}{*}{1.42} \\
\hline & $\% \mathrm{BF}$ & $0.035(0.005)$ & $<.001^{*}$ & & \\
\hline \multirow{2}{*}{ Model 2} & PACER (laps) & $-0.012(0.004)$ & $.0050^{*}$ & \multirow{2}{*}{0.45} & \multirow{2}{*}{1.49} \\
\hline & BMI $z$ score & $0.201(0.057)$ & $.0007^{*}$ & & \\
\hline
\end{tabular}

${ }^{+} P$-value for testing $H_{0}: \beta=0$ versus $H_{A}: \beta \neq 0$.

$* *$ Cross-validation Root Mean Square Error (RMSE) on original scale for $m=1000$ re-samples with sample size $n_{1}=50\left(\right.$ model fitting) and $n_{2}=32(\operatorname{model}$ validation)

$* P<.05$.

\section{Discussion}

Given that CVF is an important predictor of cardiovascular and metabolic health problems independent of weight, a method to accurately and feasibly assess childhood fitness levels in addition to BMI in larger school-based or population-based studies is needed. While a school- or field-based test by definition is easier to use in community settings, the value of school-based assessments of fitness is greatly enhanced if they have been shown to have predictive value for other health outcomes, such as IR. This study shows that the PACER is a valid school-based test of CVF and is predictive of IR, independent of body composition. Moreover, the combination of school-based measurements of both childhood fitness (PACER) and fatness (BMI $z$ score) predicts IR to a degree similar to laboratory-based measurements of fitness $\left(\mathrm{VO}_{2} \mathrm{max} / \mathrm{kgLBM}\right.$ by maximal treadmill testing) and fatness (\%BF by DXA). Thus, the PACER can be used to translate findings of laboratorybased studies to large population-based settings to identify children with low fitness levels and/or increased risk for IR. To our knowledge, this study is the first to identify a correlation between a school-based fitness test in children and measurements of IR.

This study showed that CVF, measured either by a laboratory-based or a school-based assessment, is an important predictor of insulin resistance, independent of fatness. Some previous studies have shown that laboratory-based assessment of fitness in children has a highly significant correlation with IR after controlling for body composition $[3,13-17]$, while others have not [18-20]. A previous study by our group found that $\mathrm{VO}_{2}$ max/kgLBM (by maximal treadmill testing) was significantly correlated with $\mathrm{HOMA}_{\mathrm{IR}}$ $(r=-0.42)$ [3]. In the present study, the laboratory-based assessment of fitness was a significant independent predictor of IR. The new contribution of this study is the correlation of school-based fitness tests in children with measures of IR. The PACER correlated very closely and significantly with HOMA IR $_{\text {I }}\left(r_{s}=-0.60\right)$, and after controlling for body composition, the PACER was still independently predictive of IR (Model 2, Table 3).

We compared a laboratory-based method to a schoolbased method of predicting insulin resistance using multivariate regression analysis. Model 1 utilized laboratorybased assessments of fitness and body composition while
Model 2 included school-based assessments of fitness and body composition. The laboratory-based model had an RMSE of 1.42 compared to 1.49 in the school-based model (a smaller RMSE indicates better prediction). It can be reasonably argued that this small difference in predictive value is counterbalanced by the ability to administer the school-based tests to a much larger population of children.

Another valid question is whether assessment of fitness adds substantial value to simply measuring and tracking obesity. The RMSE for BMI $z$ score is 1.59 (Table 2), compared to 1.49 for Model 2. Additionally, a "weight-only" model would not capture the many benefits of fitness testing and fitness promotion that extend beyond the prediction of IR $[4,21,22]$.

It is interesting to note that the scatterplot comparing PACER results to $\mathrm{HOMA}_{\mathrm{IR}}$ (Figure 2) shows high variability in IR at low fitness levels, and progressively less variability as the fitness levels improve. This could indicate that the increased IR variability at low fitness levels reflects individual genetic predispositions for obesity-induced IR, and that higher fitness levels can improve insulin sensitivity in all persons. Our group has previously shown that increasing fitness levels in middle school children can lead to improvements in fasting insulin levels [23], and the effect of improved fitness on IR and the risk for type 2 diabetes has been a consistent finding in adults $[4,8]$. In this cross-sectional study, the effect of improved fitness levels on IR cannot be determined.

This study has limitations. The HOMA $\mathrm{IR}$ is a useful approximation of insulin resistance measured by euglycemic clamp studies, but does have a degree of error compared with that gold standard. Correlation analysis can overestimate the relationship between variables and therefore has intrinsic limitations. Additionally, puberty can increase insulin resistance and may be a confounding variable in the relationship between fitness testing and IR. Finally, our multivariate models did not include family history of type 2 diabetes, which would likely improve the prediction of IR by accounting for a child's genetic predisposition for IR. Future studies hope to incorporate family history in order to improve the predictive value of school-based assessments for IR.

The relative contributions of fitness and fatness to the development of IR continue to be debated, and this study was not designed to address that issue. Regardless of their relative importance, it is clear that improvements in both 
fitness and fatness will yield important health consequences for increasingly obese and sedentary children [22]. Thus, it is essential to develop interventions to reduce insulin resistance that are applicable to the "real world" of children. These interventions will need a field-based metric that can be easily and broadly implemented to translate exercise laboratory study findings to the public health setting.

\section{Conclusions}

With rates of both obesity and poor fitness increasing in children, development of IR will put increasing numbers of children at risk of developing early metabolic and cardiovascular disease. Precise measurement of IR involves blood studies of insulin levels required to maintain euglycemia after glucose administration (euglycemic clamp study), an impractical procedure in school or community settings. Laboratory-based prediction of IR, through measurement of CVF (by maximal treadmill testing) and body composition (by DXA), is also resource intensive and impractical in nonlaboratory settings. This study shows that (1) the PACER is a valid school-based test of CVF and is predictive of IR; (2) simple measures of both childhood fitness (PACER) and body composition (BMI $z$ score) together accurately predict IR to a greater degree than BMI alone, approximating the degree of prediction from logistically complex and expensive laboratory tests of fitness and body composition. Thus, the PACER offers a valid school-based test of CVF that is predictive of IR and allows findings from exercise laboratory studies to be translated to a school or community setting.

\section{Acknowledgments}

The first author was supported through a grant from the Thrasher Research Fund and in part by an NIH postdoctoral training Grant (T32 DK077586). The authors thank the administration and volunteer students of River Bluff Middle School (Stoughton, WI) and Hamilton Middle School (Madison, WI) for their assistance in performing this project.

\section{References}

[1] F. S. Facchini, N. Hua, F. Abbasi, and G. M. Reaven, "Insulin resistance as a predictor of age-related diseases," The Journal of Clinical Endocrinology \& Metabolism, vol. 86, no. 8, pp. 35743578, 2001.

[2] N. Gungor, T. Hannon, I. Libman, F. Bacha, and S. Arslanian, "Type 2 diabetes mellitus in youth: the complete picture to date," Pediatric Clinics of North America, vol. 52, no. 6, pp. 1579-1609, 2005.

[3] D. B. Allen, B. A. Nemeth, R. R. Clark, S. E. Peterson, J. Eickhoff, and A. L. Carrel, "Fitness is a stronger predictor of fasting insulin levels than fatness in overweight male middleschool children," Journal of Pediatrics, vol. 150, no. 4, pp. 383387, 2007.

[4] US Department of Health and Human Services (USDHHS), Physical Activity and Health: A Report of the Surgeon General, US Department of Health and Human Services, Centers for Disease Control and Prevention, Atlanta, Ga, USA, 1996.
[5] X. Sui, M. J. LaMonte, J. N. Laditka, et al., "Cardiorespiratory fitness and adiposity as mortality predictors in older adults," Journal of the American Medical Association, vol. 298, no. 21, pp. 2507-2516, 2007.

[6] M. Wei, J. B. Kampert, C. E. Barlow, et al., "Relationship between low cardiorespiratory fitness and mortality in normal-weight, overweight, and obese men," Journal of the American Medical Association, vol. 282, no. 16, pp. 1547-1553, 1999.

[7] C. D. Lee, S. N. Blair, and A. S. Jackson, "Cardiorespiratory fitness, body composition, and all-cause and cardiovascular disease mortality in men," American Journal of Clinical Nutrition, vol. 69, no. 3, pp. 373-380, 1999.

[8] S. N. Blair and S. Brodney, "Effects of physical inactivity and obesity on morbidity and mortality: current evidence and research issues," Medicine and Science in Sports and Exercise, vol. 31, supplement 11, pp. S646-S662, 1999.

[9] G. J. Welk and M. D. Meredith, Eds., Fitnessgram/Activitygram Reference Guide, The Cooper Institute, Dallas, Tex, USA, 2008.

[10] L. S. Conwell, S. G. Trost, W. J. Brown, and J. A. Batch, "Indexes of insulin resistance and secretion in obese children and adolescents: a validation study," Diabetes Care, vol. 27, no. 2, pp. 314-319, 2004.

[11] N. Gungor, R. Saad, J. Janosky, and S. Arslanian, "Validation of surrogate estimates of insulin sensitivity and insulin secretion in children and adolescents," Journal of Pediatrics, vol. 144, no. 1, pp. 47-55, 2004.

[12] L. A. Leger, D. Mercier, C. Gadoury, and J. Lambert, "The multistage 20 metre shuttle run test for aerobic fitness," Journal of Sports Sciences, vol. 6, no. 2, pp. 93-101, 1988.

[13] J. Z. Kasa-Vubu, C. C. Lee, A. Rosenthal, K. Singer, and J. B. Halter, "Cardiovascular fitness and exercise as determinants of insulin resistance in postpubertal adolescent females," The Journal of Clinical Endocrinology \& Metabolism, vol. 90, no. 2, pp. 849-854, 2005.

[14] G. Imperatore, Y. J. Cheng, D. E. Williams, J. Fulton, and E. W. Gregg, "Physical activity, cardiovascular fitness, and insulin sensitivity among U.S. adolescents: the National Health and Nutrition Examination Survey, 1999-2002," Diabetes Care, vol. 29, no. 7, pp. 1567-1572, 2006.

[15] B. Gutin, Z. Yin, M. C. Humphries, W. H. Hoffman, B. Gower, and P. Barbeau, "Relations of fatness and fitness to fasting insulin in black and white adolescents," Journal of Pediatrics, vol. 145, no. 6, pp. 737-743, 2004.

[16] D. A. Rubin, R. G. McMurray, and J. S. Harrell, "Insulin and weight status in adolescents: independent effects of intensity of physical activity and peak aerobic power," Pediatric Exercise Science, vol. 20, no. 1, pp. 29-39, 2008.

[17] C.-Y. Ku, B. A. Gower, G. R. Hunter, and M. I. Goran, "Racial differences in insulin secretion and sensitivity in prepubertal children: role of physical fitness and physical activity," Obesity Research, vol. 8, no. 7, pp. 506-515, 2000.

[18] G. D. C. Ball, G. Q. Shaibi, M. L. Cruz, M. P. Watkins, M. J. Weigensberg, and M. I. Goran, "Insulin sensitivity, cardiorespiratory fitness, and physical activity in overweight Hispanic youth," Obesity Research, vol. 12, no. 1, pp. 77-85, 2004.

[19] B. Gutin, S. Islam, T. Manos, N. Cucuzzo, C. Smith, and M. E. Stachura, "Relation of percentage of body fat and maximal aerobic capacity to risk factors for atherosclerosis and diabetes in black and white seven- to eleven-year-old children," Journal of Pediatrics, vol. 125, no. 6, part 1, pp. 847-852, 1994. 
[20] G. Q. Shaibi, M. L. Cruz, G. D. C. Ball, et al., "Cardiovascular fitness and the metabolic syndrome in overweight Latino youths," Medicine and Science in Sports and Exercise, vol. 37, no. 6, pp. 922-928, 2005.

[21] W. B. Strong, R. M. Malina, C. J. R. Blimkie, et al., "Evidence based physical activity for school-age youth," Journal of Pediatrics, vol. 146, no. 6, pp. 732-737, 2005.

[22] "Active healthy living: prevention of childhood obesity through increased physical activity," Pediatrics, vol. 117, no. 5, pp. 1834-1842, 2006.

[23] A. L. Carrel, R. R. Clark, S. E. Peterson, B. A. Nemeth, J. Sullivan, and D. B. Allen, "Improvement of fitness, body composition, and insulin sensitivity in overweight children in a school-based exercise program: a randomized, controlled study," Archives of Pediatrics and Adolescent Medicine, vol. 159, no. 10, pp. 963-968, 2005. 